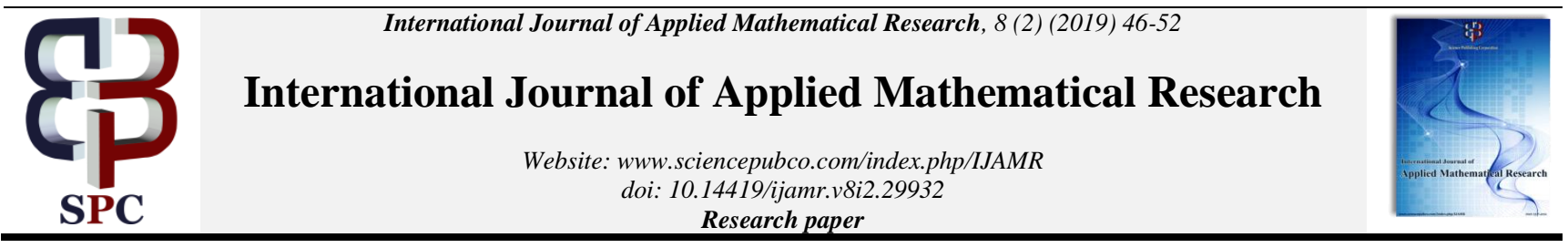

\title{
New robust-ridge estimators for partially linear model
}

\author{
Mervat M. Elgohary ${ }^{1}$, Mohamed R. Abonazel ${ }^{2 *}$, Nahed M. Helmy ${ }^{1}$, Abeer R. Azazy ${ }^{1}$ \\ ${ }^{1}$ Faculty of Commerce (Girls Branch), Al-Azhar University, Cairo, Egypt \\ ${ }^{2}$ Department of Applied Statistics and Econometrics, Faculty of Graduate Studies for Statistical Research, Cairo University, Giza, Egypt \\ *Corresponding author E-mail: mabonazel@hotmail.com
}

\begin{abstract}
This paper considers the partially linear model when the explanatory variables are highly correlated as well as the dataset contains outliers. We propose new robust biased estimators for this model under these conditions. The proposed estimators combine least trimmed squares and ridge estimations, based on the spline partial residuals technique. The performance of the proposed estimators and the Speckman-spline estimator has been examined by a Monte Carlo simulation study. The results indicated that the proposed estimators are more efficient and reliable than the Speckman-spline estimator.
\end{abstract}

Keywords: Least Trimmed Squares; Monte Carlo Simulation; Spline Smoothing; Semi-Parametric Regression; Ridge Regression; Robust Regression

\section{Introduction}

The Partially linear model (PLM) is one of the most commonly used semi-parametric regression models, which it allows both parametric and nonparametric specifications in the regression function. This model has gained great popularity since it was first introduced by Engle et al (1986) and has been widely applied in economics, social, and biological sciences. A PLM is defined by:

$y_{i}=x_{i}^{\prime} \beta+g\left(z_{i}\right)+u_{i} ; i=1,2, \ldots, n$

Where $y_{i}$ denotes the response variable, $u_{i}$ is the random error term, and $x_{i}, z_{i}$ are $p \times 1$ and $1 \times 1$ of regressors, respectively. The finite dimensional parameter $\beta$ is the parametric part of the model, and the unknown function $g(\cdot)$ is the non-parametric part of it. The classical assumptions of this model are:

1) $E\left(u_{i} \mid x_{i}, z_{i}\right)=0 \forall i=1, \ldots, n$.

2) $E\left(u_{i}^{2} \mid x_{i}, z_{i}\right)=\sigma^{2} \forall i=1, \ldots, n$.

3) $\operatorname{cov}\left(u_{i}, u_{i^{\prime}}\right)=0 \forall i \neq i^{\prime} ; i=i^{\prime}=1, \ldots, n$.

4) $\operatorname{cov}\left(u_{i}, x_{i}\right)=0$ and $\operatorname{cov}\left(u_{i}, z_{i}\right)=0 \forall i=1, \ldots, n$.

Speckman (1988) proposed a general estimation method for PLM based on the partial residuals technique (PRT), and he considered the kernel approach to estimate the nonparametric component. There are other estimators have been proposed for this model, such as Chen and Shiau (1991), Ahn and Powell (1993), Hamilton and Truong (1997), Yatchew (1997, 2000, 2003), Fadili and Bullmore (2005), Aydin (2014), Abonazel and Gad (2018), and El-Sayed et al (2019). Härdle et al (2000) and Abonazel (2018a) reviewed some of these estimators.

Recently, Abonazel et al (2019) modified the Speckman estimator by using the spline smoothing approach, and they showed that the PRT based on spline smoothing approach is more efficient than traditional PRT based on the kernel smoothing approach.

In this paper, we propose efficient estimators of the PLM if the dataset combining the problem of multicollinearity and outlier values. The proposed estimators are the modified version of Abonazel et al (2019) estimator based on a method that combines least trimmed squares (LTS) and ridge estimations. The similar estimators have been provided by Amini and Roozbeh (2016) and Roozbeh (2016) based on the kernel smoothing approach.

The rest of the paper is organized as follows. In the next section, we introduce the Speckman-Spline estimator that was proposed by Abonazel et al (2019). Our proposed estimators are presented in Section 3. While in Section 4, the Monte Carlo simulation study is conducted to compare the performance of the different estimators. Concluding remarks are included in Section 5. 


\section{Speckman-spline estimator}

The PLM in (1) can be written in matrix form as:

$y=X \beta+g(Z)+u$

Where $y=\left(y_{1}, \ldots, y_{n}\right)^{\prime}, Z=\left(z_{1}, \ldots, z_{n}\right)^{\prime}, u=\left(u_{1}, \ldots, u_{n}\right)^{\prime}$, and $X^{\prime}=\left[\begin{array}{lll}x_{1} & \cdots & x_{n}\end{array}\right]$. Taking the conditional expectation with respect to $\mathrm{Z}$ and differencing the two equations leads to (Härdle et al, 2004):

$\tilde{y}=\tilde{X} \beta+\tilde{u}$

Where $\tilde{y}=y-E(y \mid Z), \tilde{X}=X-E(X \mid Z)$, and $\tilde{u}=u-E(u \mid Z)$. Using the modified regression in (2), the vector of the parametric parameters $(\beta)$ can be estimated separately. The modified variables $\tilde{X}$ and $\tilde{y}$ are calculated using the fact that the conditional tion $E(y \mid Z)$ can be estimated through a non-parametric regression on the explanatory variable $Z$.

The Nadaraya-Watson kernel approach has been used by Speckman (1988) to estimate the nonparametric part in PLM. However, in Abonazel et al (2019) estimator, the nonparametric part has been estimated by the spline smoothing approach. Abonazel et al (2019) showed that their estimation is more efficient than the traditional Speckman estimation. We will call Abonazel et al (2019) estimator as Speckman-spline (SS) estimator, and we can summarize SS estimator in the following algorithm:

Step 1: Making a smoother spline matrix $S_{\lambda}$, depending on smoothing parameter $\lambda$ and the knot points.

Step 2: Calculating $\tilde{X}=\left(I-S_{\lambda}\right) X$ and $\widetilde{y}=\left(I-S_{\lambda}\right) y$.

Step 3: Estimating the parametric component: $\hat{\beta}=\left(\widetilde{X}^{\prime} \tilde{X}\right)^{-1} \tilde{X}^{\prime} \tilde{y}$.

Step 4: Estimating the non-parametric component: $\hat{g}_{\lambda}=S_{\lambda}(y-X \hat{\beta})=S_{\lambda} y^{*}$; where the smoothing estimate $\hat{g}_{\lambda}$ of the fitted values $y^{*}=y-X \hat{\beta}$ is projected by: $\hat{g}_{\lambda}=\left[\hat{g}_{\lambda}\left(k_{1}\right), \ldots, \hat{g}_{\lambda}\left(k_{n}\right)\right]^{\prime}=S_{\lambda}\left(y_{1}^{*}, \ldots, y_{n}^{*}\right)^{\prime}=S_{\lambda} y^{*}$, where $\hat{g}_{\lambda}$ is a natural cubic spline with knots at $\kappa_{1}, \ldots, \kappa_{n}$ for a fixed $\lambda>0$, and $S_{\lambda}$ is a well-known positive-definite matrix.

To gain better perspective on smoothing spline, the estimation of the parameters of PLM can be performed by minimizing the following sum of squares equation:

$S S(g, \hat{\beta})=\sum_{i=1}^{n}\left[\left(y_{i}-x_{i}^{\prime} \hat{\beta}\right)-g\left(z_{i}\right)\right]^{2}+\lambda \int_{a}^{b}\left[g^{\prime \prime}(z)\right]^{2} d z$

Where $\hat{\beta}$ is the estimated parametric component given from step 3 in the algorithm above. To solve Equation (3), an iterative algorithm is required.

\section{The proposed estimator}

In this paper, we consider the PLM in the case of the columns of the design matrix $X$ have a near-linear dependence, so that $X^{\prime} X$ is nearly singular. In this case, the OLS estimate becomes highly sensitive to random errors in the observed response variable with large variances. This case called in econometric literature as multicollinearity problem. There are many methods to handle this problem in regression models such as ridge, Liu, principal components, etc.

Besides multicollinearity problem, we consider the dataset contain outlier values. The outliers are another common problem in the regression analysis. There are many robust regression methods that are used to overcome the effects of outliers.

In this paper, we propose a new robust biased estimator of PLM based on LTS method plus ridge regression. We use LTS-ridge estimator in stage 3 (in the above algorithm) instead of OLS estimator, the formula of the proposed (LTS-ridge) estimator is (Kan et al, 2013):

$\hat{\beta}_{l t s-r}=\left(\tilde{X}^{\prime} \tilde{X}+k_{l t s} I\right)^{-1}\left(\tilde{X}^{\prime} \tilde{X}\right) \hat{\beta}_{l t s}$

Where $k_{l t s}$ is the robust choice of the k parameter in ridge regression, and the total MSE (TMSE) of $\hat{\beta}_{l t s-r}$ is

$\operatorname{TMSE}\left(\hat{\beta}_{l t s-r}\right)=\sum_{a=1}^{p}\left[\left(\sigma^{2} \delta_{a}+k_{l t s}^{2} \beta^{\prime} \beta\right) /\left(\delta_{a}+k_{l t s}\right)^{2}\right]$

Where $\delta_{a}$ values are the eigenvalues of $\tilde{X}^{\prime} \tilde{X}$ matrix. This estimator is resistant to the combined problem of multicollinearity and outliers. Here, $\beta$ and $\sigma^{2}$ are replaced by their LTS estimates $\hat{\beta}_{l t s}$ and $\hat{\sigma}_{l t s}^{2}$ to obtain the minimum TMSE estimate, see Arslan and Billor (1996). In this paper, we suggest using the three ridge parameters in $\hat{\beta}_{l t s-r}$ :

1) Updating the formula of the ridge parameter that is proposed by Kibria (2003):

$\hat{k}_{l t s}($ Kibria $)=\operatorname{median}\left(\frac{1}{\widehat{\alpha}_{j}^{2}}\right)$

2) We suggest the following new ridge parameters:

$\hat{k}_{l t s}($ new 1$)=\left[\hat{k}_{l t s}(\text { Kibria })\right]^{1 / p}\left[\max \left(\frac{1}{\hat{\alpha}_{j}^{2}}\right)\right]^{1 / p}$

$\hat{k}_{l t s}(\operatorname{new} 2)=\left[\operatorname{sum}\left(\frac{1}{\widehat{\alpha}_{j}^{2}}\right)\right]^{1 / p}\left[\max \left(\frac{1}{\widehat{\alpha}_{j}^{2}}\right)\right]^{1 / p}$

Where $\widehat{\alpha}=\left(\Phi^{\prime} \tilde{X}^{\prime} \tilde{X} \Phi\right)^{-1} \Phi^{\prime} \widetilde{X}^{\prime} \tilde{y}$; with $\Phi$ eigenvectors of $\tilde{X}^{\prime} \tilde{X}$ matrix. Using the three ridge parameters above in $\hat{\beta}_{\text {lts }-r}$, we get three robust-ridge (RR) estimators: RR1, RR2, and RR3, respectively. 


\section{The simulation study}

In this section, we investigate the performance of the presented estimators above through a Monte Carlo simulation study. In fact, we make a comparison study between the SS estimator and the proposed robust-ridge (RR1, RR2, and RR3) estimators. R software is used to perform our simulation study. For information about how to make Monte Carlo simulation studies using R, see Abonazel (2018b).

The simulated dataset is carried out based on Equation (1) with the following simulation settings:

1) The different sample sizes have been used as: $n=100,150,200,300$, and 500.

2) The number of parametric coefficients are $p=2,8$; with $\beta^{\prime} \beta=1$ and $\beta_{1}=\cdots=\beta_{p}$, as Månsson and Shukur (2011) and KaÇiranlar and Dawoud (2018).

3) Two functions have been used for the nonparametric component in the model:

$g_{1}=1.5 \sin (\pi z)$ and $g_{2}=1.5 \sin \left(\pi z^{2}\right)$.

4) The explanatory variables: $X \sim M V N\left(1, \Sigma_{X}\right)$, where diag $\left(\Sigma_{x}\right)=1$ and off-diag $\left(\Sigma_{x}\right)=\rho_{x}$, as Abonazel and Farghali (2018) and Abonazel (2019), where $\rho_{x}=0.75$ and 0.95 . While the variable $Z$ is generated from uniform distribution from -1 to 1 .

5) The errors are generated from normal distribution with mean zero and standard deviation $\sigma=0.5$. To generate some outlier values in the model with $\tau=15 \%$ or $\tau=35 \%$ ratios, we replace some values randomly (according to the selected ratio) from the vector of errors with other values generated from uniform distribution from 3 to 7.

6) All Monte Carlo experiments involved 1000 replications and all the results of all separate experiments are obtained by precisely the same series of random numbers.

The goodness of fit of $\hat{g}$ and $\hat{\beta}$ can be quantified by computing the average of mean squared error (AMSE) values of $\hat{g}$ and $\hat{\beta}$ at each iteration $l ; l=1, \ldots, 1000$. The MSE of $\hat{g}$ and $\hat{\beta}$ are calculated as:

$M S E_{l}(\hat{g})=\frac{1}{n} \sum_{i=1}^{n}\left[\hat{g}\left(z_{i}\right)-g\left(z_{i}\right)\right]^{2} ; M S E_{l}(\hat{\beta})=\frac{1}{p} \sum_{a=1}^{p}\left[\hat{\beta}_{a}-\beta_{a}\right]^{2}$

Where $\hat{g}\left(z_{i}\right)$ and $\hat{\beta}_{a}$ are the estimated values of $g\left(z_{i}\right)$ and $\beta_{a}$, respectively. To simplify the tables of the simulation results, we presented the total AMSE (TAMSE): TAMSE = AMSE of parametric part + AMSE of nonparametric part.

The simulation results are recorded in Tables 1-8. These tables present the TAMSE of the estimators in different factors $\left(p, \rho_{x}\right.$, and nonparametric function). Specifically, Tables 1-4 present the TAMSE values of the estimators when $p=2$ (with $\rho_{x}=0.75,0.95$ and $g_{1}, g_{2}$ ), while case of $p=8$ (with the same cases of $\rho_{x}$ and the same nonparametric functions) is presented in Tables 5-8.

From Tables 1-8, we can summarize some effects for all estimators in the following points:

- As $\tau$ increases, the TAMSE values increase.

- As $n$ increases, the TAMSE values decrease.

- As $\rho_{x}$ increases, the TAMSE values increase.

- As $p$ increases, the TAMSE values increase.

In general, in all simulation situations, we can conclude that the TAMSE values of all RR estimators are smaller than the TAMSE values of SS estimator, and the efficient RR estimators are RR1 and RR3.

Table 1: TAMSE Values for the Estimators of $g_{1}$ when $p=2$ and $\rho_{x}=.75$

\begin{tabular}{|c|c|c|c|c|}
\hline$n$ & \multicolumn{3}{|c|}{ RR Estimators } & RR3 \\
\hline \multicolumn{5}{|c|}{$\boldsymbol{\tau}=15 \%$} \\
\hline 100 & 0.7908 & 0.5438 & 0.6196 & 0.7090 \\
\hline 150 & 0.6849 & 0.4685 & 0.5450 & 0.6345 \\
\hline 200 & 0.6636 & 0.4934 & 0.5713 & 0.6619 \\
\hline 300 & 0.6241 & 0.4365 & 0.5093 & 0.5970 \\
\hline 500 & 0.5979 & 0.4647 & 0.5442 & 0.6353 \\
\hline \multicolumn{5}{|c|}{$\boldsymbol{\tau}=35 \%$} \\
\hline 150 & 3.2041 & 0.8933 & 0.9733 & 1.0658 \\
\hline 200 & 3.2143 & 0.9071 & 0.9862 & 1.0821 \\
\hline 300 & 3.1619 & 0.7888 & 0.8763 & 0.9752 \\
\hline 500 & 3.1231 & 0.7500 & 0.8468 & 0.9562 \\
\hline
\end{tabular}

Table 2: TAMSE Values for the Estimators of $g_{1}$ when $p=2$ and $\rho_{x}=.95$

\begin{tabular}{|c|c|c|c|c|}
\hline$n$ & \multicolumn{3}{|c|}{ RR Estimators } & RR3 \\
\hline \multicolumn{5}{|c|}{$\boldsymbol{\tau}=15 \%$} \\
\hline 100 & 1.1643 & 0.3515 & 0.3797 & 0.4027 \\
\hline 150 & 0.9231 & 0.3027 & 0.3368 & 0.3707 \\
\hline 200 & 0.8345 & 0.2417 & 0.2689 & 0.2999 \\
\hline 300 & 0.7367 & 0.2457 & 0.2746 & 0.3090 \\
\hline 500 & 0.6671 & 0.2240 & 0.2577 & 0.2972 \\
\hline \multicolumn{5}{|c|}{$\boldsymbol{\tau}=35 \%$} \\
\hline 150 & 3.5555 & 1.0405 & 1.0579 & 1.0601 \\
\hline 200 & 3.4909 & 0.8579 & 0.8826 & 0.8981 \\
\hline 300 & 3.3505 & 0.7054 & 0.7327 & 0.7581 \\
\hline 500 & 3.2201 & 0.5665 & 0.6000 & 0.6380 \\
\hline
\end{tabular}


Table 3: TAMSE Values for the Estimators of $g_{2}$ when $p=2$ and $\rho_{x}=.75$

\begin{tabular}{|c|c|c|c|c|}
\hline $\boldsymbol{n}$ & \multicolumn{3}{|c|}{ RR Estimators } & RR3 \\
\hline \multicolumn{5}{|c|}{$\boldsymbol{\tau}=15 \%$} \\
\hline 100 & 0.7879 & 0.5359 & 0.6101 & 0.6956 \\
\hline 150 & 0.6851 & 0.5151 & 0.5919 & 0.6787 \\
\hline 200 & 0.6630 & 0.5302 & 0.6127 & 0.7060 \\
\hline 300 & 0.6234 & 0.4515 & 0.5283 & 0.6187 \\
\hline 500 & 0.5980 & 0.4524 & 0.5316 & 0.6233 \\
\hline \multicolumn{5}{|c|}{$\boldsymbol{\tau}=35 \%$} \\
\hline 150 & 3.2031 & 0.9638 & 1.0450 & 1.1348 \\
\hline 200 & 3.2173 & 0.8819 & 0.9625 & 1.0528 \\
\hline 300 & 3.1605 & 0.7921 & 0.8734 & 0.9677 \\
\hline 500 & 3.1266 & 0.7063 & 0.7948 & 0.8984 \\
\hline
\end{tabular}

Table 4: TAMSE Values for the Estimators of $g_{2}$ when $p=2$ and $\rho_{x}=.95$

\begin{tabular}{|c|c|c|c|c|}
\hline $\boldsymbol{n}$ & \multicolumn{3}{|c|}{ RR Estimators } & RR3 \\
\hline \multicolumn{5}{|c|}{$\boldsymbol{\tau}=15 \%$} \\
\hline 100 & 1.1573 & 0.3660 & 0.3924 & 0.4137 \\
\hline 150 & 0.9214 & 0.3086 & 0.3423 & 0.3757 \\
\hline 200 & 0.8335 & 0.2646 & 0.2988 & 0.3376 \\
\hline 300 & 0.7357 & 0.2494 & 0.2853 & 0.3269 \\
\hline 500 & 0.6677 & 0.2109 & 0.2427 & 0.2789 \\
\hline \multicolumn{5}{|c|}{$\boldsymbol{\tau}=35 \%$} \\
\hline 150 & 3.5547 & 1.0784 & 1.0977 & 1.1029 \\
\hline 200 & 3.4935 & 0.8534 & 0.8733 & 0.8873 \\
\hline 300 & 3.3498 & 0.7082 & 0.7413 & 0.7748 \\
\hline 500 & 3.2229 & 0.5219 & 0.5647 & 0.6123 \\
\hline
\end{tabular}

Table 5: TAMSE Values for the Estimators of $g_{1}$ when $p=8$ and $\rho_{x}=.75$

\begin{tabular}{|c|c|c|c|c|}
\hline$n$ & \multicolumn{3}{|c|}{ RR Estimators } & RR3 \\
\hline \multicolumn{5}{|c|}{$\boldsymbol{\tau}=15 \%$} \\
\hline 100 & 0.8510 & 0.1602 & 0.1425 & 0.1338 \\
\hline 150 & 0.7232 & 0.1353 & 0.0862 & 0.0829 \\
\hline 200 & 0.7041 & 0.1161 & 0.0661 & 0.0626 \\
\hline 300 & 0.6481 & 0.0975 & 0.0410 & 0.0389 \\
\hline 500 & 0.6159 & 0.0902 & 0.0230 & 0.0222 \\
\hline \multicolumn{5}{|c|}{$\boldsymbol{\tau}=35 \%$} \\
\hline 100 & 3.5408 & 1.4267 & 1.5154 & 1.4008 \\
\hline 150 & 3.2972 & 0.8792 & 0.9280 & 0.8722 \\
\hline 200 & 3.3195 & 0.6905 & 0.7272 & 0.6975 \\
\hline 300 & 3.1821 & 0.4612 & 0.4808 & 0.4679 \\
\hline 500 & 3.1375 & 0.3183 & 0.3019 & 0.2980 \\
\hline
\end{tabular}

Table 6: TAMSE Values for the Estimators of $g_{1}$ when $p=8$ and $\rho_{x}=.95$

\begin{tabular}{|c|c|c|c|c|}
\hline \multirow[b]{2}{*}{$n$} & \multicolumn{4}{|c|}{ RR Estimators } \\
\hline & $\mathrm{SS}$ & RR1 & RR2 & RR3 \\
\hline \multicolumn{5}{|c|}{$\boldsymbol{\tau}=15 \%$} \\
\hline 100 & 1.4964 & 0.2451 & 0.2690 & 0.2029 \\
\hline 150 & 1.1292 & 0.1567 & 0.1933 & 0.1466 \\
\hline 200 & 1.0161 & 0.1130 & 0.1568 & 0.1231 \\
\hline 300 & 0.8300 & 0.0694 & 0.1073 & 0.0860 \\
\hline 500 & 0.7315 & 0.0435 & 0.0739 & 0.0616 \\
\hline \multicolumn{5}{|c|}{$\boldsymbol{\tau}=35 \%$} \\
\hline 100 & 4.6042 & 2.1915 & 1.9073 & 1.5595 \\
\hline 150 & 3.9774 & 1.3846 & 1.3212 & 1.1045 \\
\hline 200 & 3.7422 & 1.0338 & 1.0322 & 0.8859 \\
\hline 300 & 3.5109 & 0.6925 & 0.7299 & 0.6433 \\
\hline 500 & 3.3413 & 0.4413 & 0.4885 & 0.4488 \\
\hline
\end{tabular}

Table 7: TAMSE Values for the Estimators of $g_{2}$ when $p=8$ and $\rho_{x}=.75$

\begin{tabular}{|c|c|c|c|c|}
\hline$n$ & SS & RR Estimators & RR2 & RR3 \\
\hline \multicolumn{5}{|c|}{$\boldsymbol{\tau}=15 \%$} \\
\hline 100 & 0.8514 & 0.1668 & 0.1425 & 0.1344 \\
\hline 150 & 0.7260 & 0.1295 & 0.0873 & 0.0827 \\
\hline 200 & 0.7047 & 0.1083 & 0.0672 & 0.0656 \\
\hline 300 & 0.6489 & 0.1204 & 0.0415 & 0.0398 \\
\hline 500 & 0.6155 & 0.0791 & 0.0235 & 0.0230 \\
\hline \multicolumn{5}{|c|}{$\boldsymbol{\tau}=35 \%$} \\
\hline 100 & 3.5354 & 1.4131 & 1.5005 & 1.3976 \\
\hline 150 & 3.2920 & 0.8780 & 0.9393 & 0.8962 \\
\hline 200 & 3.3133 & 0.6807 & 0.7239 & 0.6940 \\
\hline 300 & 3.1785 & 0.4582 & 0.4806 & 0.4681 \\
\hline
\end{tabular}




\begin{tabular}{|c|c|c|c|c|}
\hline 500 & 3.1376 & 0.3074 & 0.2993 & 0.2959 \\
\hline \multicolumn{5}{|c|}{ Table 8: TAMSE Values for the Estimators of $g_{2}$ when $p=8$ and $\rho_{x}=.95$} \\
\hline & & RR Esti & & \\
\hline$n$ & SS & RR1 & RR2 & RR3 \\
\hline \multicolumn{5}{|l|}{$\boldsymbol{\tau}=15 \%$} \\
\hline 100 & 1.5234 & 0.2499 & 0.2640 & 0.1962 \\
\hline 150 & 1.1496 & 0.1499 & 0.1920 & 0.1504 \\
\hline 200 & 1.0186 & 0.1206 & 0.1633 & 0.1287 \\
\hline 500 & 0.7365 & 0.0438 & 0.0729 & 0.0618 \\
\hline \multicolumn{5}{|l|}{$\boldsymbol{\tau}=35 \%$} \\
\hline 100 & 4.5575 & 2.0993 & 1.8432 & 1.5063 \\
\hline 150 & 3.9534 & 1.3338 & 1.2723 & 1.0695 \\
\hline 200 & 3.8058 & 1.0273 & 1.0282 & 0.8809 \\
\hline 300 & 3.5059 & 0.6777 & 0.7175 & 0.6351 \\
\hline 500 & 3.3261 & 0.4126 & 0.4564 & 0.4184 \\
\hline
\end{tabular}

Graphically, we will illustrate the degree of goodness of fit of the four estimators for the several nonparametric functions $\left(g_{1}, g_{2}, g_{3}=\right.$ $\left.3 \sin \left(\pi z^{3}\right), g_{4}=z+z^{2}+z^{4}\right)$ via the simulated datasets with different factors $\left(\tau, n, p\right.$, and $\left.\rho_{x}\right)$. Figures 1-4 show the fitted curves of the estimators based on the four nonparametric functions, respectively. From Figure 1, we find that the fitted curves based on RR estimators are closer to the true curve than SS estimator; although the model contains many outliers and the explanatory variables are correlated. The same results can be concluded from figures 2-4. This means that RR estimators perform better regardless of the form of the nonparametric function and are not sensitive to outliers in the model.

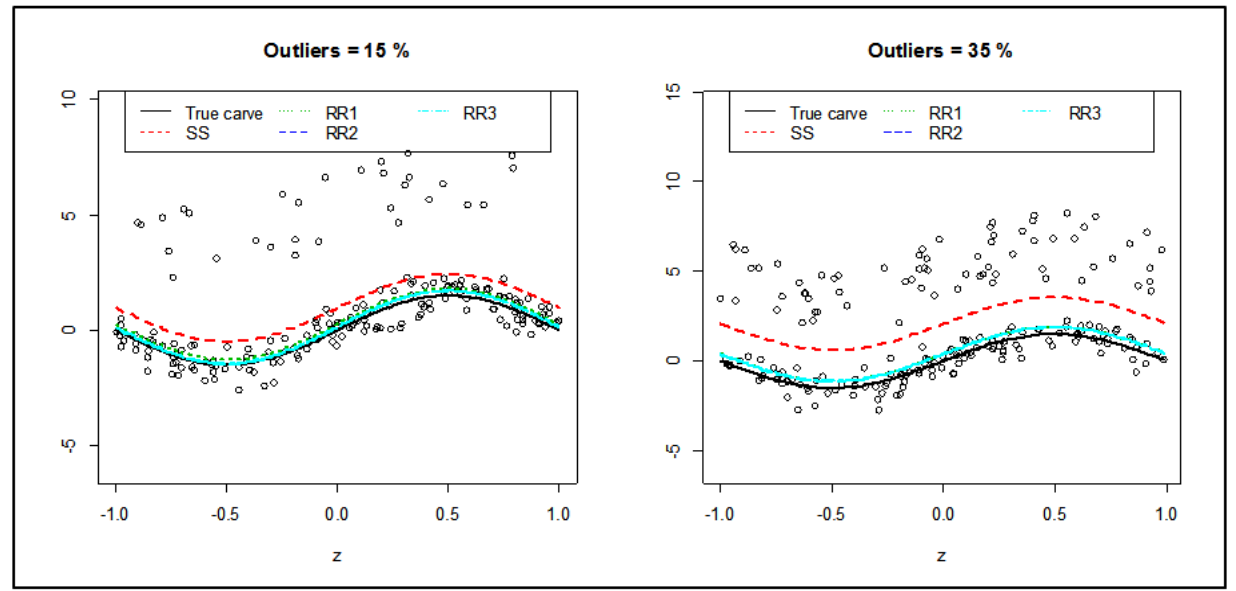

Fig. 1: Fitted Values for the Estimators of $g_{1}$ when $n=200, p=4$, and $\rho_{\mathrm{x}}=.85$

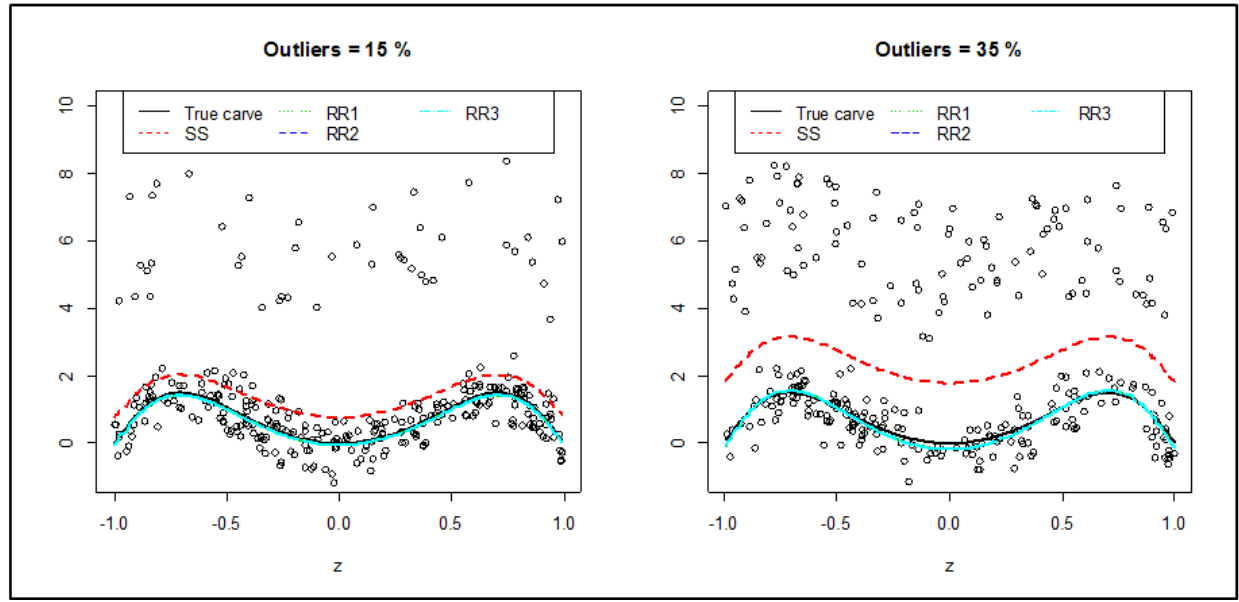

Fig. 2: Fitted Values for the Estimators of $g_{2}$ when $n=300, p=8$, and $\rho_{x}=.95$. 


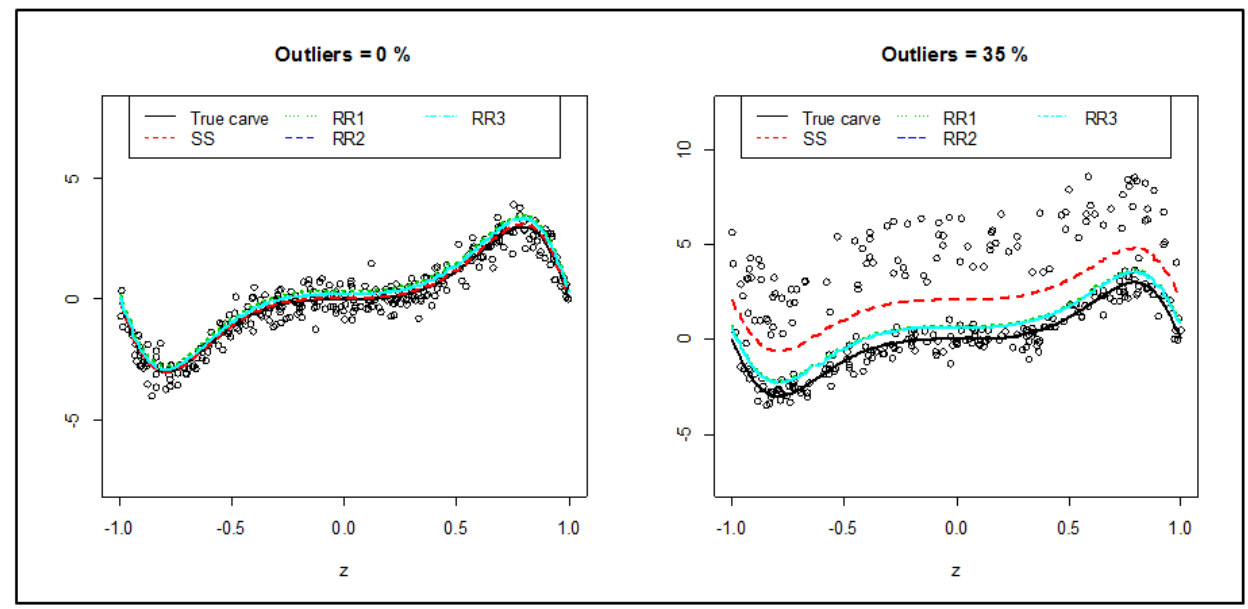

Fig. 3: Fitted Values for the Estimators of $g_{3}$ when $n=300, p=8$, and $\rho_{x}=0$.

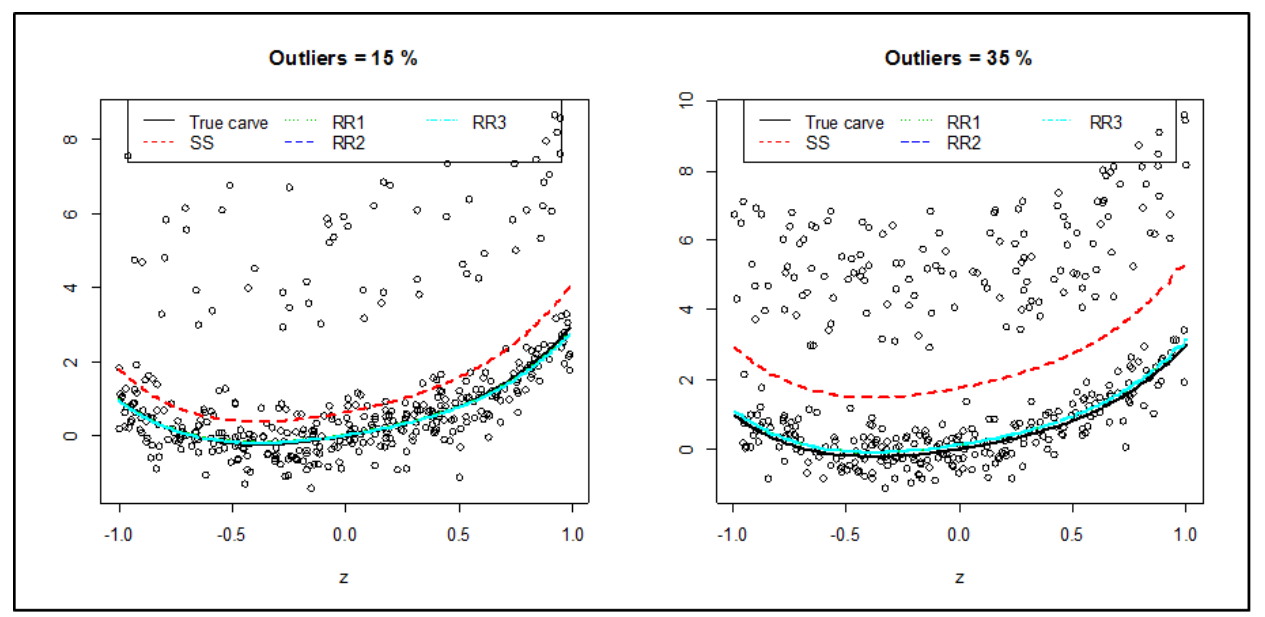

Fig. 4: Fitted Values for the Estimators of $g_{4}$ when $n=400, p=6$, and $\rho_{x}=0.95$.

\section{Conclusion}

In this paper, we developed new LTS-ridge estimators for PLM when there are high inter-correlations between the explanatory variables as well as the dataset contains outliers. Moreover, new biasing parameters are suggested. A Monte Carlo simulation study was conducted to evaluate the performance of SS estimator (proposed by Abonazel et al, 2019) and our LTS-ridge estimators. The simulation results indicate that our LTS-ridge estimators are efficient than SS estimator in all situations.

\section{References}

[1] Abonazel, M. R. (2018a). Different estimation methods of partially linear regression models. Working paper. Faculty of Graduate Studies for Statistical Research, Cairo University, Egypt.

[2] Abonazel, M. R. (2018b). A practical guide for creating Monte Carlo simulation studies using R. International Journal of Mathematics and Computational Science, 4(1), 18-33.

[3] Abonazel, M. R. (2019). New ridge estimators of SUR model when the errors are serially correlated. International Journal of Mathematical Archive, 10(7):53-62.

[4] Abonazel, M. R. \& Farghali, R. A. (2018). Liu-type multinomial logistic estimator. Sankhya B: The Indian Journal of Statistics. https://doi.org/10.1007/s13571-018-0171-4.

[5] Abonazel, M. R., \& Gad, A. A. (2018). Robust partial residuals estimation in semiparametric partially linear model. Communications in StatisticsSimulation and Computation, 1-14. https://doi.org/10.1080/03610918.2018.1494279.

[6] Abonazel, M. R., Helmy, N. \& Azazy, A. (2019). The performance of Speckman estimation for partially linear model using kernel and spline smoothing approaches. International Journal of Mathematical Archive, 10(6):10-18.

[7] Ahn, H., \& Powell, J. L. (1993). Semiparametric estimation of censored selection models with a nonparametric selection mechanism. Journal of Econometrics, 58(1-2), 3-29. https://doi.org/10.1016/0304-4076(93)90111-H.

[8] Amini, M., \& Roozbeh, M. (2016). Least trimmed squares ridge estimation in partially linear regression models. Journal of Statistical Computation and Simulation, 86(14):2766-2780. https://doi.org/10.1080/00949655.2015.1128433.

[9] Arslan, O., \& Billor, N. (1996). Robust ridge regression estimation based on the GM-estimators. Journal of Mathematical and Computational Science, $9(1), 1-9$.

[10] Aydin, D. (2014). Estimations of the partially linear models with smoothing spline based on different selection methods: a comparative study. Pakistan Journal of Statistics, 30(1), 35-56.

[11] Chen, H., \& Shiau, J. J. H. (1991). A two-stage spline smoothing method for partially linear models. Journal of Statistical Planning and Inference, 27(2), 187-201. https://doi.org/10.1016/0378-3758(91)90015-7.

[12] El-Sayed, S. M., Abonazel, M. R., \& Seliem, M. M. (2019), B-Spline Speckman Estimator of Partially Linear Model. Working paper. Faculty of Graduate Studies for Statistical Research, Cairo University, Egypt. 
[13] Engle, R. F., Granger, C. W., Rice, J., \& Weiss, A. (1986). Semiparametric estimates of the relation between weather and electricity sales. Journal of the American statistical Association, 81(394), 310-320. https://doi.org/10.1080/01621459.1986.10478274.

[14] Fadili, J. M., \& Bullmore, E. (2005). Penalized partially linear models using sparse representations with an application to fMRI time series. IEEE Transactions on signal processing, 53(9), 3436-3448. https://doi.org/10.1109/TSP.2005.853207.

[15] Hamilton, S. A., \& Truong, Y. K. (1997). Local linear estimation in partly linear models. Journal of Multivariate Analysis, 60(1), 1-19. https://doi.org/10.1006/jmva.1996.1642.

[16] Härdle, W., Liang, H., \& Gao, J. (2000). Partially linear models. Springer Science \& Business Media. https://doi.org/10.1007/978-3-642-57700-0.

[17] Härdle, W. K., Müller, M., Sperlich, S., \& Werwatz, A. (2004). Nonparametric and semiparametric models. Springer Science \& Business Media. https://doi.org/10.1007/978-3-642-17146-8.

[18] Kaçıranlar, S., \& Dawoud, I. (2018). On the performance of the Poisson and the negative binomial ridge predictors. Communications in StatisticsSimulation and Computation, 47(6):1751-1770. https://doi.org/10.1080/03610918.2017.1324978.

[19] Kan, B., Alpu, Ö, \& Yazıc1, B. (2013) robust ridge and robust Liu estimator for regression based on the LTS estimator. Journal of Applied Statistics, 40(3):644-655. https://doi.org/10.1080/02664763.2012.750285.

[20] Kibria, B. G. (2003). Performance of some new ridge regression estimators. Communications in Statistics-Simulation and Computation, 32(2):419435. https://doi.org/10.1081/SAC-120017499.

[21] Månsson, K., \& Shukur, G. (2011). On ridge parameters in logistic regression. Communications in Statistics-Simulation and Computation, 40:3366-3381. https://doi.org/10.1080/03610926.2010.500111.

[22] Roozbeh, M. (2016). Robust ridge estimator in restricted semiparametric regression models. Journal of Multivariate Analysis, 147:127-144. https://doi.org/10.1016/j.jmva.2016.01.005.

[23] Speckman, P. (1988). Kernel smoothing in partial linear models. Journal of the Royal Statistical Society: Series B (Methodological), 50(3), 413436. https://doi.org/10.1111/j.2517-6161.1988.tb01738.x,

[24] Yatchew, A. (1997). An elementary estimator of the partial linear model. Economics Letters, 57(2), 135-143. https://doi.org/10.1016/S01651765(97)00218-8.

[25] Yatchew, A. (2000). Scale economies in electricity distribution: a semiparametric analysis. Journal of Applied Econometrics, 15(2), 187-210. https://doi.org/10.1002/(SICI)1099-1255(200003/04)15:2<187::AID-JAE548>3.0.CO;2-B.

[26] Yatchew, A. (2003). Semiparametric regression for the applied econometrician. Cambridge University Press. https://doi.org/10.1017/CBO9780511615887. 